\title{
Normas DRIS multivariadas para avaliação do estado nutricional de laranjeira 'Pera' no estado do Amazonas ${ }^{1}$
}

\author{
Multivariate DRIS standards for the assessment of the nutritional status of the Pera \\ orange in the state of Amazonas
}

\author{
Jairo Rafael Machado Dias ${ }^{2 *}$, Paulo Guilherme Salvador Wadt ${ }^{3}$, Carlos Alberto Franco Tucci ${ }^{4}$, José Zilton Lopes \\ Santos $^{4}$ e Silvio Vieira da Silva ${ }^{4}$
}

\begin{abstract}
RESUMO - Para utilização da diagnose da composição nutricional ou do sistema integrado de diagnose e recomendação de relações multivariadas (DRIS) na avaliação do estado nutricional de laranjeira, faz-se necessário a definição de valores de referência que sejam adequados para refletir suas condições nutricionais. Neste trabalho, objetivou-se estabelecer normas DRIS multivariadas e avaliar seu desempenho comparativamente aos padrões nutricionais definidos pela literatura na avaliação do estado nutricional de laranjeiras 'Pera' no estado do Amazonas. Avaliou-se o estado nutricional de 120 glebas comerciais de laranjeira 'Pera' enxertadas em limoeiro 'Cravo', sendo que os padrões de referência foram definidos a partir do conjunto das glebas. O diagnóstico nutricional pelo método DRIS conjugado a utilização de normas genéricas comparativamente aos padrões nutricionais propostos pela literatura apresentou baixo grau de concordância na avaliação do estado nutricional de laranjeira 'Pera' no estado do Amazonas.
\end{abstract}

Palavras-chave: Análise foliar. Citrus limonia. Citrus sinensis. Plantas-nutrição. Relações nutricionais.

\begin{abstract}
In order to use nutritional-composition diagnosis or the diagnosis and integrated recommendation system of multivariate relationships (DRIS) when assessing the nutritional status of the orange, it becomes necessary to define reference values that are suitable for indicating nutritional conditions. This study aimed to establish multivariate DRIS standards, and to evaluate their comparative performance in assessing the nutritional status of the Pera orange in the state of Amazonas against nutritional standards as defined in the literature. We evaluated the nutritional status of 120 commercial plots of a hybrid of the Pera orange and Rangpur lime, the reference values being defined from the group of plots. DRIS standards for nitrogen $(\mathrm{N})$, phosphorus $(\mathrm{P})$, potassium $(\mathrm{K})$, calcium $(\mathrm{Ca})$, magnesium (Mg), sulfur (S), boron $(\mathrm{B})$, copper $(\mathrm{Cu})$, iron $(\mathrm{Fe})$, manganese $(\mathrm{Mn})$ and zinc $(\mathrm{Zn})$ were established and nutritional diagnosis by DRIS, combined with the comparative use of generic standards to those proposed in the literature, showed a low level of agreement when assessing the nutritional status of the Pera orange in the state of Amazonas.
\end{abstract}

Key words: Foliar analysis. Citrus limonia. Citrus sinensis. Nutrition-plant. Nutritional relationships.

\footnotetext{
*Autor para correspondência

${ }^{1}$ Recebido para publicação em 06/04/2012; aprovado em 13/10/2012

Parte da Tese de Doutorado do primeiro autor, apresentada ao Programa de Pós-Graduação em Agronomia Tropical da Universidade Federal do Amazonas/UFAM

${ }^{2}$ Universidade Federal de Rondônia/UNIR, Avenida Norte Sul, 7300, Rolim de Moura-RO, Brasil, 76.940-000, jairorafaelmdias@ hotmail.com ${ }^{3}$ Embrapa Acre, Rodovia BR 364 km 14, Caixa Postal 321, Rio Branco-AC, Brasil, 69.900-056, paulogswadt@dris.com.br

${ }^{4}$ Universidade Federal do Amazonas/UFAM, Avenida General R. O. J. Ramos, 3000, Campus Universitário, Manaus-AM, Brasil, 69.077-000, ctucci@ ufam.edu.br, ziltton@yahoo.com.br e silviovieiras@hotmail.com
} 


\section{INTRODUÇÃO}

No cenário mundial, o Brasil destaca-se como principal produtor de laranja, com participação superior a $80 \%$ no comércio internacional de suco concentrado congelado. Os valores da exportação deste produto, junto com outros derivados, têm gerado mais de 1,8 bilhões de dólares para o setor citrícola brasileiro (NEVES et al., 2010). Dentre as cultivares comerciais, a Pera destaca-se por atender tanto as exigências da indústria quanto ao mercado in natura. (BREMER NETO et al., 2008; QUAGGIO et al., 2011; SANTANA et al., 2007).

Apesar de a citricultura brasileira ser competitiva, o rendimento tem sido baixo (aproximadamente $11 \mathrm{t} \mathrm{ha}^{-1}$ ) no Estado do Amazonas quando comparado ao Estado de São Paulo $\left(26 \mathrm{t} \mathrm{ha}^{-1}\right)$ (INSTITUTO BRASILEIRO GEOGRAFIA E ESTATÍSTICA, 2011). No Amazonas, a produção concentra-se nos municípios de Manaus e circunvizinhos, entre eles, Iranduba, Rio Preto da Eva, Manacapuru e Presidente Figueiredo (INSTITUTO BRASILEIRO DE GEOGRAFIAE ESTATÍSTICA, 2011). Nessa região, a citricultura, apesar de bem adaptada às condições edafoclimáticas locais, carece de informações que permitam o manejo adequado das adubações, que se agrava pela insuficiência de práticas conservacionistas para o manejo do solo (COELHO; NASCIMENTO, 2002).

Uma alternativa para aprimorar o manejo das adubações consiste no monitoramento nutricional das glebas, que em espécies cultivadas comercialmente tem sido frequentemente realizado pelos métodos do nível crítico (NC) ou das faixas de suficiência (FS). Por estes métodos comparam-se teores foliares nutricionais de uma gleba comercial com padrões de referência obtidos de plantas sadias e de elevada produtividade, normalmente disponibilizados pela literatura (KURIHARA et al., 2005). Como exemplo, tem-se os padrões nutricionais disponíveis para glebas de citros cultivados no estado de São Paulo (MALAVOLTA et al., 1994; QUAGGIO et al., 2005). Entretanto, o método das FS apresenta dependência de calibração local, o que reduz a eficácia do diagnóstico quando se extrapola para condições distintas do local de origem destas referências nutricionais (FAGERIA et al., 2009).

Para diminuir a dependência da calibração local e melhorar a qualidade dos diagnósticos obtidos foi proposto o sistema integrado de diagnose e recomendação (DRIS) (BEAUFILS, 1973). Dentre as variações das funções DRIS existe a utilização de relações multivariadas entre os nutrientes, mais conhecido como método da diagnose da composição nutricional-CND (PARENT, 2011). Esse método tem sido frequentemente utilizado com sucesso na diagnose do estado nutricional em cultivos comerciais (ANJANEYULU;RAGHUPATHI,2010;MAGALLANESQUINTANAR et al., 2004; SERRA et al., 2010).
O uso do DRIS requer também o desenvolvimento de padrões nutricionais, neste caso denominados de normas DRIS. Estas normas devem ser representativas de toda diversidade de condições de manejo e edafoclimáticas da gleba cultivada (BEAUFILS, 1973). Neste sentido, objetivou-se estabelecer normas DRIS multivariadas e avaliar seu desempenho comparativamente aos padrões nutricionais definidos pela literatura na avaliação do estado nutricional de laranjeiras 'Pera' no estado do Amazonas.

\section{MATERIAL E MÉTODOS}

Cento e vinte glebas comerciais de laranjeiras 'Pera' enxertadas em limoeiro 'Cravo' foram monitoradas quanto ao estado nutricional entre os meses de fevereiro de 2010 a abril de 2011. As glebas selecionadas apresentavam densidade de 208 a 408 plantas ha $^{-1}$, idade entre 8 a 15 anos e tamanho médio de 1,0 ha, sendo representativos da região produtora de citros no Amazonas, em cinco municípios: Iranduba ( $03^{\circ} 17^{\prime} 06^{\prime \prime} \mathrm{S}$ e $\left.60^{\circ} 11^{\prime} 09^{\prime \prime} \mathrm{W}\right)$, Manacapuru $\left(03^{\circ} 17^{\prime} 59^{\prime \prime} \mathrm{S}\right.$ e $\left.60^{\circ} 37^{\prime} 14^{\prime \prime} \mathrm{W}\right)$; Manaus $\left(03^{\circ} 06^{\prime} 00^{\prime \prime} \mathrm{S}\right.$ e $\left.60^{\circ} 01^{\prime} 00^{\prime \prime} \mathrm{W}\right)$, Presidente Figueiredo $\left(02^{\circ} 01^{\prime} 02^{\prime \prime} \mathrm{S}\right.$ e $60^{\circ} 01^{\prime} 30^{\prime \prime}$ W) e Rio Preto da Eva $\left(02^{\circ} 41^{\prime} 56^{\prime \prime} \mathrm{S}\right.$ e $\left.59^{\circ} 42^{\prime} 00^{\prime \prime} \mathrm{W}\right)$.

O clima da região é classificado como tropical úmido - Af (Köppen), com temperatura média anual de $26^{\circ} \mathrm{C}$ e precipitação pluvial média de $2.550 \mathrm{~mm}_{\text {ano }}{ }^{-1}$. O período chuvoso está compreendido entre os meses de dezembro até abril. No primeiro trimestre do ano observa-se o maior acúmulo de chuvas. O período mais quente fica compreendido entre agosto e outubro (SISTEMA DE PROTEÇÃO DA AMAZÔNIA, 2005).

Em cada gleba monitorada foram amostradas aleatoriamente 25 árvores, correspondendo a um total de 100 folhas recém-amadurecidas, coletadas na terceira posição de lançamento a partir do ápice de ramos, com fruto de seis meses de idade e diâmetro entre $2-4 \mathrm{~cm}$, na face das árvores referentes aos quatro pontos cardeais e sempre na altura mediana da planta (MALAVOLTA et al., 1994). O material vegetal coletado foi acondicionado em sacos de papel e transportado para o laboratório, onde foram lavados, secados, moídos e submetidas às analises dos teores totais $\mathrm{N}, \mathrm{P}, \mathrm{K}, \mathrm{Ca}, \mathrm{Mg}, \mathrm{S}, \mathrm{B}, \mathrm{Cu}, \mathrm{Fe}, \mathrm{Mn}$ e Zn, empregando-se os métodos sugeridos por Malavolta et al. (1997).

Calculou-se o valor do complemento dos nutrientes para o total da biomassa da folha, denominado de valor R, empregando-se a Equação 1:

$R=100-(v N+v P+v K+v C a+v M g+v S+v B+v C u+v F e+v M n+v Z n)$

onde, $\mathrm{R}$ representa o conteúdo da matéria e a massa correspondente aos demais nutrientes não avaliados 
e, $v N, v P, v K, v C a, v M g, v S, v B, v C u, v F e, v M n$ e $v Z n$ são os teores de N, P, K, Ca, Mg, S, B, Cu, Fe, Mn e Zn, respectivamente, expressos em dag $\mathrm{kg}^{-1}$.

A média geométrica ( $\mathrm{mGeo}$ ) dos teores nutricionais (expressos em dag $\mathrm{kg}^{-1}$ ) foi calculada para cada amostra foliar, empregando-se a Equação 2:

$m G e o=(v N . v P . v K . v C a . v M g . v S . v B . v C u . v F e . v M n . v Z n \cdot R)^{(I / 2)}$

Para cada nutriente, determinou-se sua respectiva variável multinutriente $(z X)$ pelo logarítimo neperiano do quociente entre $v X$ e a média geométrica (mGeo) dos teores nutricionais, empregando-se a Equação 3:

$z X=\ln (v X / m G e o)$

onde, $z X$ representa o valor da relação multivariada de cada um dos nutrientes avaliados $(z N, z P, z K, z C a, z M g$, $z S, z B, z C u, z F e, z M n$ e $z Z n)$.

Com os valores das relações multivariadas de cada gleba, calculou-se para o conjunto da população monitorada os parâmetros descritivos: média aritmética $(m X)$, desvio padrão $(s X)$ e coeficiente de variação, determinando-se as normas DRIS de relações multivariadas. A utilização de todo o conjunto de dados para a obtenção das normas DRIS traz como benefícios maior representatividade e melhor estimativa dos parâmetros populacionais (BEVERLY, 1993).

Os índices DRIS multivariados para cada nutriente na respectiva gleba, foram calculados pela relação multivariada log-centrada (PARENT, 2011), empregando-se a Equação 4:
$I \_X=(Z x-m X) / s X$

onde, $I \_X, m X$ e $s X$ representam o índice DRIS de relação multivariada, a norma média e a norma desvio padrão para os nutrientes $\mathrm{N}, \mathrm{P}, \mathrm{K}, \mathrm{Ca}, \mathrm{Mg}, \mathrm{S}, \mathrm{B}, \mathrm{Cu}$, $\mathrm{Fe}, \mathrm{Mn}$ e Zn.

Calculou-se também o índice de balanço nutricional médio (IBNm), empregando-se a Equação 5:

$$
\begin{aligned}
& I B N m=\left(\left|I \_N /+\right| I \_P /+\left|I \_K /+\right| I \_C a /+\mid I \_M g /+\right. \\
& \left.\left|I \_S /+\right| I \_B|+| I \_C u|+| I F e|+| I \_M n|+| I \_Z n \mid\right) 11
\end{aligned}
$$

O nutriente foi considerado nutricionalmente equilibrado quando seu índice DRIS em módulo foi menor que o IBNm. Insuficiente quando seu índice DRIS em módulo foi simultaneamente maior que o IBNm e menor que zero. E, em excesso nutricional ou consumo de luxo quando seu índice DRIS em módulo foi maior que o IBNm e maior que zero, simultaneamente (WADT, 2005).

Paralelamente, para cada nutriente obteve-se também avaliação do estado nutricional das glebas monitoradas, utilizando-se do método das FS propostas pela literatura para efeito comparativo com os diagnósticos proporcionados pelo DRIS. As FS propostas pela literatura (MALAVOLTA et al., 1994; QUAGGIO et al., 2005) estão contidas na Tabela 1. Estas faixas foram adotadas, por serem utilizadas rotineiramente na região do presente estudo, como também em outros estados brasileiros (BOARETTO et al., 2007; FERNANDES et al., 2010; SANTANA et al., 2008).

Tabela 1 - Faixas de suficiência nutricional definidas pela literatura para avaliação do estado nutricional de laranjeiras em fase de produção

\begin{tabular}{lcccccc}
\hline & \multicolumn{5}{c}{ Quaggio et al. (2005) } & \multicolumn{3}{c}{ Malavolta et al. (1994) } \\
\cline { 2 - 7 } Nutrientes & \multicolumn{5}{c}{$\mathrm{g} \mathrm{kg}^{-1}$} \\
\cline { 2 - 7 } & Insuficiente & Equilibrado & Excesso & Insuficiente & Equilibrado & Excesso \\
\hline $\mathrm{N}$ & $<23$ & $23-27$ & $>27$ & $<24$ & $24-26$ & $>30$ \\
$\mathrm{P}$ & $<1,2$ & $1,2-1,6$ & $>1,6$ & $<1,2$ & $1,2-1,7$ & $>2,9$ \\
$\mathrm{~K}$ & $<10$ & $10-15$ & $>15$ & $<10$ & $10-14$ & $>14$ \\
$\mathrm{Ca}$ & $<35$ & $35-45$ & $>45$ & $<35$ & $35-40$ & $>40$ \\
$\mathrm{Mg}$ & $<3$ & $3-4$ & $>4$ & $<2,5$ & $2,5-3$ & $>3$ \\
$\mathrm{~S}$ & $<2$ & $2-3$ & $>3$ & $<2$ & $2-2,5$ & $>2,5$ \\
\hline & & & $\mathrm{mg} \mathrm{kg}^{-1}$ & & $>140$ \\
$\mathrm{~B}$ & $<50$ & $50-100$ & $>100$ & $<60$ & $60-140$ & $>140$ \\
$\mathrm{Cu}$ & $<4$ & $4,1-10$ & $>10$ & $<10$ & $10-30$ & $>30$ \\
$\mathrm{Fe}$ & $<49$ & $50-120$ & $>120$ & $<130$ & $130-300$ & $>300$ \\
$\mathrm{Mn}$ & $<34$ & $35-50$ & $>50$ & $<25$ & $25-49$ & $>49$ \\
$\mathrm{Zn}$ & $<34$ & $35-50$ & $>50$ & $<25$ & $25-49$ & $>49$ \\
\hline
\end{tabular}


Para interpretação dos teores nutricionais nas glebas monitoradas, adotaram-se três estados nutricionais, sendo: i) Equilibrado, quando a concentração do nutriente no tecido foliar se encontra entre o intervalo definido como ideal para cultura. ii) Insuficiente, quando a concentração do nutriente no tecido foliar se encontra abaixo do intervalo definido como ideal para cultura. iii) Excesso nutricional ou consumo de luxo, quando a concentração do nutriente no tecido foliar se encontra acima do intervalo definido como ideal para cultura.

A frequência de distribuição das glebas monitoradas em relação a cada estado nutricional, diagnosticadas pelo DRIS e pelas FS, foram contrastadas entre si pelo teste qui quadrado $\left(\chi^{2}\right)$, ao nível de $1 \%$ de significância. Os diagnósticos das glebas monitoradas foram comparados quanto ao grau de concordância relativo a cada método de avaliação (DRIS e FS), computando-se para cada gleba e nutriente, os casos de concordância. Foram considerados concordantes quando dois diferentes métodos ou duas faixas distintas de suficiência resultaram no mesmo diagnóstico, expressando-se os resultados em percentagem de diagnósticos corretos.

Quantificou-se ainda, o número de vezes que os nutrientes apresentaram índices DRIS multivariados mais negativo e mais positivo, nas glebas, a partir das normas DRIS estabelecidas neste trabalho. O cálculo das normas, funções, índices DRIS multivariados e teste quiquadrado $\left(\chi^{2}\right)$ foram realizados em planilha eletrônica. As análises estatísticas descritivas: mínimo, máximo, média, desvio padrão, coeficientes de curtose, simetria e variação pelo programa estatístico Assistat versão 6.2 (SILVA; AZEVEDO, 2002).

\section{RESULTADOS E DISCUSSÃO}

$\mathrm{Ca}, \mathrm{N}$ e K, nesta ordem são requeridos em maiores quantidades nos tecidos foliares de laranjeiras 'Pera', enquanto que $\mathrm{P}, \mathrm{Mg}$ e $\mathrm{S}$ são exigidos praticamente na mesma ordem de grandeza $\left(0,8\right.$ a $\left.6,5 \mathrm{~g} \mathrm{~kg}^{-1}\right)$ (Tabela 2). A concentração de $\mathrm{Ca}$, particularmente em folhas de citros, já foi relatada por Quaggio et al. (2005) ser superior a dos outros nutrientes, incluindo $\mathrm{N}$ em todos os tecidos, com exceção dos frutos.

Os coeficientes de curtose foram negativos para $\mathrm{K}$ e $\mathrm{S}$, indicando que a distribuição é mais achatada que a normal (platicúrtica). A curtose é uma medida do grau de achatamento de uma distribuição em relação à curva normal. Do modo semelhante, o coeficiente de simetria foi negativo para N. A simetria é uma medida da forma de distribuição dos dados quanto à distribuição da curva normal, indicando neste caso, que a cauda desta curva é viezada à esquerda (FREITAS et al., 2008). Observa-se também maior coeficiente de variação $(\mathrm{CV})$ para os teores foliares de $\mathrm{Fe}, \mathrm{Cu}$ e $\mathrm{Mg}$, nesta ordem e menor CV para N (Tabela 2). Quando se pretende estimar FS a partir da população que deu origem às normas DRIS, infere-se que quanto maior for o $\mathrm{CV}$, proporcionalmente será a amplitude da faixa crítica (PARTELLI et al., 2006a).

Para o estabelecimento das normas DRIS multivariadas optou-se pela utilização do conjunto de dados da população, conforme já proposto para cupuaçueiros (DIAS et al., 2010a; DIAS et al., 2010b; WADT et al., 2011) e mangueiras (WADT; SILVA, 2010). Nestas normas, valores positivos ou negativos indicam apenas o valor da média aritmética das relações

Tabela 2 - Mínimo (Mín.), máximo (Máx.), média, desvio padrão (DP), coeficientes de curtose (CC), simetria (CS) e variação (CV) das concentrações de N, P, K, Ca, Mg, S, B, Cu, Fe, Mn e Zn nas 120 glebas de laranjeiras 'Pera' monitoradas no estado do Amazonas

\begin{tabular}{lrrrrrrr}
\hline \multicolumn{1}{c}{ Nutrientes } & Mín. & Máx. & Média & DP & CC & CS & CV (\%) \\
\hline $\mathrm{N}\left(\mathrm{g} \mathrm{kg}^{-1}\right)$ & 10,0 & 36,0 & 29,1 & 3,3 & 8,6 & $-1,92$ & 11,5 \\
$\mathrm{P}\left(\mathrm{g} \mathrm{kg}^{-1}\right)$ & 1,0 & 2,9 & 1,7 & 0,3 & 1,1 & 0,88 & 20,3 \\
$\mathrm{~K}\left(\mathrm{~g} \mathrm{~kg}^{-1}\right)$ & 2,0 & 15,2 & 7,5 & 3,3 & $-1,1$ & 0,04 & 44,4 \\
$\mathrm{Ca}\left(\mathrm{g} \mathrm{kg}^{-1}\right)$ & 15,6 & 48,1 & 28,6 & 6,9 & 0,1 & 0,68 & 24,4 \\
$\mathrm{Mg}\left(\mathrm{g} \mathrm{kg}^{-1}\right)$ & 1,5 & 6,5 & 4,1 & 2,1 & 64,6 & 6,89 & 50,2 \\
$\mathrm{~S}\left(\mathrm{~g} \mathrm{~kg}^{-1}\right)$ & 0,8 & 3,3 & 1,8 & 0,5 & $-0,1$ & 0,58 & 26,2 \\
$\mathrm{~B}\left(\mathrm{mg} \mathrm{kg}^{-1}\right)$ & 15,5 & 115,7 & 51,1 & 17,9 & 1,0 & 0,59 & 34,9 \\
$\mathrm{Cu}\left(\mathrm{mg} \mathrm{kg}^{-1}\right)$ & 2,0 & 77,2 & 14,2 & 16,3 & 6,3 & 2,65 & 114,9 \\
$\mathrm{Fe}\left(\mathrm{mg} \mathrm{kg}^{-1}\right)$ & 42,3 & 381,7 & 105,5 & 135,2 & 51,7 & 7,01 & 128,1 \\
$\mathrm{Mn}\left(\mathrm{mg} \mathrm{kg}^{-1}\right)$ & 3,83 & 29,0 & 13,26 & 4,78 & 0,9 & 0,89 & 36,1 \\
$\mathrm{Zn}\left(\mathrm{mg} \mathrm{kg}^{-1}\right)$ & 9,2 & 43,7 & 16,56 & 5,75 & 5,3 & 2,10 & 35,3 \\
\hline
\end{tabular}


Tabela 3 - Normas DRIS multivariadas log centradas (média, desvio padrão e coeficiente de variação) para N, P, K, Ca, Mg, S, B, Cu, $\mathrm{Fe}, \mathrm{Mn} \mathrm{Zn}$ e complemento da matéria seca (MS) para laranjeira 'Pera' no estado do Amazonas

\begin{tabular}{|c|c|c|c|}
\hline Norma DRIS & Média & $\mathrm{DP}$ & $\mathrm{CV}(\%)$ \\
\hline $\mathrm{mN}$ & 3,42 & 0,15 & 4,46 \\
\hline $\mathrm{mP}$ & 0,57 & 0,23 & 39,93 \\
\hline $\mathrm{mK}$ & 1,96 & 0,49 & 24,89 \\
\hline $\mathrm{mCa}$ & 3,39 & 0,26 & 7,57 \\
\hline $\mathrm{mMg}$ & 1,41 & 0,37 & 26,07 \\
\hline $\mathrm{mS}$ & 0,65 & 0,21 & 32,77 \\
\hline $\mathrm{mB}$ & $-2,98$ & 0,35 & 11,91 \\
\hline $\mathrm{mCu}$ & $-4,54$ & 0,66 & 14,51 \\
\hline $\mathrm{mFe}$ & $-2,37$ & 0,47 & 19,75 \\
\hline $\mathrm{mMn}$ & $-4,32$ & 0,33 & 7,55 \\
\hline $\mathrm{mZn}$ & $-4,08$ & 0,23 & 5,56 \\
\hline mMS & 6,89 & 0,12 & 1,75 \\
\hline$\sum \mathrm{mX}$ & 0 & - & - \\
\hline
\end{tabular}

multivariadas, não havendo nenhuma relação com o estado nutricional das glebas monitoradas (Tabela 3).

A utilização de normas DRIS genéricas, como as obtidas neste trabalho, tem sido questionada por diversos autores. Partelli et al. (2006b) ao compararem normas DRIS específicas entre si no diagnóstico nutricional de cafeeiros orgânicos e convencionais, indicam especificidade das normas DRIS. Esse entendimento tem sido compartilhado por inúmeros outros autores, os quais têm desenvolvimento normas DRIS específicas para determinada região ou locais (ARIZALETA et al., 2002; BARBOSA et al., 2006; LANA et al., 2010).

Por outro lado, Dias et al. (2010a) ao avaliarem normas DRIS genéricas, obtidas de pomares de cupuaçueiros cultivados em sistemas agroflorestais e em monocultivo, observaram diagnósticos semelhantes aos obtidos por normas específicas. Dias et al. (2010b) sugerem que para fruteiras na Amazônia as normas DRIS sejam genéricas, pois a busca por normas específicas pode inviabilizar o sistema, devido à grande diversidade de condições. Destaca-se que as normas DRIS precisam ser representativas da população a ser diagnosticada (BEAUFILS, 1973). E, quanto maior o número de dados envolvidos na obtenção dos padrões de referência, melhor será a representatividade da norma DRIS junto às plantas a serem diagnosticadas.

A distribuição das frequências entre as classes nutricionais (insuficiente, equilibrado e excesso) a partir dos resultados deste trabalho foram distintas para todos os nutrientes quando contrastadas às FS propostas pela literatura. Entretanto, quando comparou-se entre si as FS definidas pela literatura, a proporção de glebas insuficientes, equilibradas e com excesso nutricional foram semelhantes para N, K, S e Mn (Tabela 4).

As semelhanças nas frequências entre as classes nutricionais, utilizando-se FS definidas pela literatura para N, K, S e Mn justifica-se pelas condições edafoclimáticas semelhantes, nas quais os padrões nutricionais foram obtidos. Destaca-se que o estabelecimento de padrões nutricionais regionais, como os obtidos neste trabalho é particularmente importante, pois o diagnóstico nutricional torna-se mais confiável quando comparado a teores críticos não regionalizados. A determinação de normas DRIS locais possibilita ainda, a determinação de FS a partir da sua população de referência, conforme já obtido para outras culturas, destacando-se café (FARNEZI et al., 2009; PARTELLI et al., 2006a), soja (URANO et al., 2007), algodão (SERRA et al., 2010), arroz (GUINDANI et al., 2009) e eucalipto (WADT et al., 1998).

Comparando-se cada um dos diagnósticos obtidos para cada nutriente pelo método DRIS em relação às FS da literatura, verifica-se que o grau de concordância foi baixo para maioria dos nutrientes. Por outro lado, contrastando entre si as FS da literatura, observa-se que para N, P, K, Ca, S, B, Mn e Zn o grau de concordância foi superior a $80 \%$ (Tabela 5).

Ainda, o diagnóstico pelo DRIS em comparação a FS proposta por Malavolta et al. (1994) mostraram maior grau de concordância para $\mathrm{P}$ e menor para Zn. Já para o contraste do DRIS em relação à FS definida por Quaggio 
Tabela 4 - Frequência em que as glebas monitoradas apresentaram estado de deficiência, equilíbrio e excesso nutricional, de acordo com a avaliação do estado nutricional de plantas de laranjeira 'Pera', pelo uso do DRIS de relações multivariadas (este trabalho) e por faixas de suficiência descritas por Malavolta et al. (1994) (GPACC) e Quaggio et al. (2005) (SP)

-Freqüência (\%)-

Métodos de diagnóstico

$\begin{array}{llll} & & & \\ & \text { Equilíbrio } & \text { Excesso } & \text { Qui-quadrado }\end{array}$

\begin{tabular}{|c|c|c|c|c|}
\hline \multicolumn{5}{|c|}{ Nitrogênio } \\
\hline Este trabalho & 16 & 60 & 24 & $215 *(2)$ \\
\hline GPACC & 3 & 12 & 85 & $292 *(3)$ \\
\hline SP & 2 & 20 & 68 & $4^{\text {ns (4) }}$ \\
\hline \multicolumn{5}{|c|}{ Fósforo } \\
\hline Este trabalho & 23 & 57 & 20 & $167 *(2)$ \\
\hline GPACC & 3 & 59 & 37 & $141 *(3)$ \\
\hline SP & 3 & 39 & 58 & $18^{*(4)}$ \\
\hline \multicolumn{5}{|c|}{ Potássio } \\
\hline Este trabalho & 28 & 38 & 34 & $1127 *(2)$ \\
\hline GPACC & 75 & 22 & 3 & $361 *(3)$ \\
\hline SP & 75 & 24 & 1 & $4^{\mathrm{ns}(4)}$ \\
\hline \multicolumn{5}{|c|}{ Cálcio } \\
\hline Este trabalho & 26 & 49 & 25 & $258^{*(2)}$ \\
\hline GPACC & 81 & 12 & 7 & $198 *(3)$ \\
\hline SP & 80 & 17 & 3 & $7 * *(4)$ \\
\hline \multicolumn{5}{|c|}{ Magnésio } \\
\hline Este trabalho & 20 & 50 & 30 & $22 *(2)$ \\
\hline GPACC & 9 & 9 & 82 & $233 *(3)$ \\
\hline SP & 19 & 30 & 51 & $39 *(4)$ \\
\hline \multicolumn{5}{|c|}{ Enxofre } \\
\hline Este trabalho & 22 & 59 & 19 & $377 *(2)$ \\
\hline GPACC & 67 & 32 & 1 & $377 *(3)$ \\
\hline SP & 67 & 32 & 1 & $0^{\operatorname{ns}(4)}$ \\
\hline \multicolumn{5}{|c|}{ Boro } \\
\hline Este trabalho & 22 & 55 & 23 & $499 *(2)$ \\
\hline GPACC & 72 & 27 & 1 & $548 *(3)$ \\
\hline SP & 49 & 50 & 1 & $21^{*(4)}$ \\
\hline \multicolumn{5}{|c|}{ Cobre } \\
\hline Este trabalho & 18 & 64 & 18 & $42 *(2)$ \\
\hline GPACC & 65 & 24 & 11 & $105^{*(3)}$ \\
\hline SP & 5 & 60 & 35 & $738 *(4)$ \\
\hline
\end{tabular}


Continuação da Tabela 4

\begin{tabular}{lcccc}
\hline & & Ferro & \\
\hline Este trabalho & 22 & 69 & 9 & $124^{*(2)}$ \\
GPACC & 93 & 5 & 3 & $898^{*(3)}$ \\
SP & 3 & 87 & 10 & $2784^{*(4)}$ \\
\hline Este trabalho & Manganês & 22 & $3752^{*(2)}$ \\
GPACC & 20 & 58 & 1 & $2070^{*(3)}$ \\
SP & 97 & 2 & 1 & $1^{\text {ns(4) }}$ \\
\hline Este trabalho & 98 & 1 & 19 & $2066^{*(2)}$ \\
GPACC & 21 & Zinco & $666^{*(3)}$ \\
SP & 90 & 60 & 1 & $25^{*(4)}$ \\
\hline
\end{tabular}

ns - Não significativo. *e ** - Significativo pelo teste de Qui-quadrado, a 5 e $1 \%$, respectivamente. ${ }^{2}$ - (Este trabalho Vs. SP). ${ }^{3}$ - (GPCAC Vs. Este trabalho). ${ }^{4}$ - (GPCAC Vs. SP)

Tabela 5 - Grau de concordância, em percentagem, entre os diagnósticos de N, P, K, Ca, Mg, S, B, Cu, Fe, Mn e Zn obtidos pelo uso do DRIS de relações multivariadas e por faixas de suficiência nutricional (Malavolta et al. 1994; Quaggio et al., 2005) em 120 glebas de laranjeiras 'Pera' no estado do Amazonas

\begin{tabular}{lccc}
\hline & \multicolumn{3}{c}{ Diagnóstico comparativo entre métodos de interpretação de análise foliar } \\
\cline { 2 - 4 } & DRIS Vs. Malavolta & DRIS Vs. Quaggio & Malavolta Vs. Quaggio \\
\cline { 2 - 4 } & 35 & 41 & 91 \\
$\mathrm{n}$ & 62 & 49 & 81 \\
$\mathrm{~K}$ & 37 & 35 & 96 \\
$\mathrm{Ca}$ & 33 & 29 & 95 \\
$\mathrm{Mg}$ & 43 & 62 & 59 \\
$\mathrm{~S}$ & 37 & 39 & 94 \\
$\mathrm{~B}$ & 41 & 52 & 81 \\
$\mathrm{Cu}$ & 46 & 67 & 16 \\
$\mathrm{Fe}$ & 26 & 76 & 7 \\
$\mathrm{Mn}$ & 18 & 18 & 98 \\
Zn & 7 & 7 & 93 \\
\hline Média & 35 & 43 & 74 \\
\hline
\end{tabular}

et al. (2005), a maior e menor concordância entre os diagnósticos foram para $\mathrm{Fe}$ e $\mathrm{Zn}$, respectivamente. Wadt et al. (2011) avaliando entre si três métodos de diagnose na avaliação do estado nutricional de cupuaçueiros, observaram alto grau de concordância entre duas funções
DRIS. Entretanto a frequência nos casos de insuficiência foi aleatória para maioria dos nutrientes, sugerindo que a elevada concordância entre diagnósticos produzidos por distintos métodos pode não implicar, necessariamente em uma avaliação adequada para o estado nutricional. 
Como o índice DRIS avalia o quanto determinado nutriente afeta no desempenho nutricional da planta à medida que se distancia do valor zero (WALWORTH; SUMNER, 1987) na Tabela 6, verifica-se que os elementos comíndices DRIS mais negativos nas glebas seguiram a ordem $\mathrm{K}>\mathrm{P}=\mathrm{B}>\mathrm{Mn}>\mathrm{Mg}>\mathrm{Cu}=\mathrm{Fe}>\mathrm{S}>\mathrm{Ca}>\mathrm{N}>\mathrm{Zn}$ e, os nutrientes com índices DRIS mais positivos, em ordem decrescente foram $\mathrm{K}>\mathrm{S}>\mathrm{Zn}>\mathrm{Cu}>=\mathrm{Mg}>\mathrm{Mn}>\mathrm{Ca}>\mathrm{B}>\mathrm{P}>\mathrm{Fe}>\mathrm{N}$.

Tabela 6 - Frequência de nutrientes com índice DRIS mais negativo (+ NE) e mais positivo (+ PO), nas 120 glebas de laranjeiras 'Pera' monitoradas no estado do Amazonas, utilizando normas DRIS multivariadas na avaliação do estado nutricional

\begin{tabular}{lrc}
\hline \multirow{2}{*}{ Nutrientes } & \multicolumn{2}{c}{ Freqüência } \\
\cline { 2 - 3 } $\mathrm{N}$ & $+\mathrm{NE}$ & $+\mathrm{PO}$ \\
$\mathrm{P}$ & 5 & 2 \\
$\mathrm{~K}$ & 15 & 6 \\
$\mathrm{Ca}$ & 22 & 22 \\
$\mathrm{Mg}$ & 7 & 10 \\
$\mathrm{~S}$ & 12 & 13 \\
$\mathrm{~B}$ & 8 & 18 \\
$\mathrm{Cu}$ & 15 & 7 \\
$\mathrm{Fe}$ & 9 & 13 \\
$\mathrm{Mn}$ & 9 & 4 \\
$\mathrm{Zn}$ & 14 & 11 \\
\hline
\end{tabular}

É interessante destacar que independente do método utilizado para o diagnóstico nutricional (DRIS e FS), o $\mathrm{K}$ foi apontado frequentemente sob desequilíbrio nutricional, tanto por insuficiência ou excesso nutricional (Tabela 4). Isto sugere relavância para o K no manejo da adubação, principalmente por ser um elemento responsivo à sua aplicação em outras regiões (MATTOS JUNIOR et al., 2010; QUAGGIO et al., 2011; QUAGGIO et al., 2006).

A ausência de adubações equilibradas nestas glebas, pode estar causando uma situação generalizada de deficiência de $\mathrm{K}$ nestes solos, de modo a refletir nas normas DRIS. Situação semelhante já foi evidenciada por Dias et al. (2011) para Pem pomares de cupuaçueiros na região amazônica. Nesse sentido, a norma DRIS para $\mathrm{K}$ (Tabela 3) poderia ser superior e, consequentemente, a proporção de plantas insuficientes poderia ser maior que a apresentada neste trabalho.

\section{CONCLUSÕES}

1. Em mais de $50 \%$ das glebas monitoradas, os nutrientes $\mathrm{K}, \mathrm{Ca}, \mathrm{Mg}, \mathrm{B}$, Mn e Zn são, nesta ordem, os elementos que se encontram em maior desequilíbrio nutricional por deficiência ou excesso a partir de normas genéricas com o uso do método DRIS de relações multivariadas;

2. No método DRIS de relações multivariadas conjugado a utilização de normas genéricas apresenta baixo grau de concordância em contraste às faixas críticas propostas pela literatura concernente à avaliação do estado nutricional de laranjeira 'Pera' na Amazônia central.

\section{AGRADECIMENTOS}

Ao Projeto PI-Citros/FAPEAM pela ajuda financeira.

\section{REFERÊNCIAS}

ANJANEYULU, K.; RAGHUPATHI, H. B. Multivariate diagnosis of nutrient imbalance in rose grown under open and protected conditions using compositional nutrient diagnosis and principal component analysis. Indian Journal of Agricultural Sciences, v. 80, n. 12, p. 1068-1061, 2010.

ARIZALETA, M. et al. Relación de los índices DRIS, índices de balance de nutrientes, contenido foliar de nutrientes y el rendimiento del cafeto em Venezuela. Bioagro, v. 14, n. 1, p. 153-159, 2002.

BARBOSA, D. H. S. G. et al. Estabelecimento de normas DRIS e diagnostico nutricional do cafeeiro arábica na região noroeste do Estado do Rio de Janeiro. Ciência Rural, v. 36, n. 1, p. 1717-1722, 2006.

BEAUFILS, E. R. Diagnosis and recommendation integrated system (DRIS). Bloemfontein: University of Natal, 1973. 132 p.

BEVERLY, R. B. DRIS Diagnoses of soybean nitrogean, phosphorus, and potassium status are usatisfactory. Journal of Plant Nutrition, v. 16, n. 1, p. 1431-1447, 1993.

BOARETTO, R. M. et al. Acúmulo de nutrientes e destino do nitrogênio $\left({ }^{15} \mathrm{~N}\right)$ aplicado em pomar jovem de laranjeira. Revista Brasileira de Fruticultura, v. 29, n. 3, p. 600-605, 2007.

BREMER NETO, H. et al. Estado nutricional e produção de laranjeira 'Pêra' em função da vegetação intercalar e cobertura morta. Pesquisa Agropecuária Brasileira, v. 43, n. 1, p. 25-35, 2008.

COElHo, T. da S.; NASCIMENTO, H. G. do. Citricultura no Amazonas: problemas, potencial produtivo e qualidade dos frutos. Cruz das Almas: Embrapa mandioca e Fruticultura, 2002. 2 p. (Citros em foco, 26).

DIAS, J. R. M. et al. Potencial de resposta à adubação para N, $\mathrm{P}, \mathrm{K}, \mathrm{Ca}$ e $\mathrm{Mg}$ em cupuaçueiros avaliados por diferentes normas DRIS. Acta Amazônica, v. 41, n. 1, p. 77-82, 2011. 
DIAS, J. R. M. et al. Normas DRIS para cupuaçueiro cultivado em monocultivo e em sistemas agroflorestais. Pesquisa Agropecuária Brasileira, v. 45, n. 1, p. 64-71, 2010a.

DIAS, J. R. M. et al. Estabelecimento de normas DRIS para o cupuaçueiro na região amazônica. Revista Caatinga, v. 23, n. 4, p. 121-128, 2010b.

FAGERIA, N. K. et al. Foliar fertilization of crop plants. Journal of Plant Nutrition, v. 32, n. 1, p. 1044-1064, 2009.

FARNESI, M. de M. et al. Diagnose nutricional de cafeeiros da região do Alto Jequitinhonha (Mg): normas DRIS e faixas críticas de nutrientes. Revista Brasileira de Ciência do Solo, v. 33, n. 1, p. 969-978, 2009.

FERNANDES, A. R. et al. Estado nutricional de pomares de laranjeira submetidos a diferentes manejos do solo. Revista de Ciências Agrárias, v. 53, n. 1, p. 52-58, 2010.

FREITAS, R. de F. et al. Técnicas de análises exploratórias em dados de cultivares de alfafa. Revista Brasileira de Zootecnia, v. 37, n. 9, p. 1531-1536, 2008.

GUINDANI, R. H. P. et al. DRIS na avaliação do estado nutricional do arroz irrigado por inundação. Revista Brasileira de Ciência do Solo, v. 22, n. 1, p. 109-118, 2009.

INSTITUTOBRASILEIRODE GEOGRAFIAEESTATÍSTICA. Produção agrícola estadual 2010: culturas permanentes. Rio de Janeiro: IBGE. 91 p. Disponível em: <http://www.ibge.gov. br/estadosat/temas.php?sigla=am\&tema=lavourapermanente 20 10>. Acesso em: 04 mar. 2012.

KURIHARA, C. H. et al. Interpretação de resultados de análise foliar. Dourados: Embrapa Agropecuária Oeste; Colombo: Embrapa Floresta, 2005. 42 p. (Documentos, 74).

LANA, R. M. Q. et al. Levantamento do estado nutricional de plantas de Coffea arabica L. pelo DRIS, na região do Alto Paranaíba - Minas Gerais. Revista Brasileira de Ciência do Solo, v. 34, n.1, p. 1147-1156, 2010.

MAGALlANES-QUINTANAR, R. et al. Compositional Nutrient Diagnosis in Nopal (Opuntia ficus-indica). Journal of the Professional Association for Cactus Development, v. 6, n. 1 p. 78-89, 2004.

MALAVOLTA, E. et al. Seja o doutor dos seus citros. Piracicaba: Potafós, 1994. 22 p. (Informações Agronômicas, 65).

MALAVOLTA, E. et al. Avaliação do estado nutricional das plantas: princípios e aplicações. Piracicaba: Potafos, 1997. 319 p.

MATTOS JUNIOR, D. et al. Soil nutrient availability and its impacto $\mathrm{n}$ fruit quality of tahiti acid lime. Revista Brasileira de Fruticultura, v. 32, n. 1, p. 335-342, 2010.

NEVES, M. F. et al. O retrato da citricultura brasileira. Ribeirão Preto: CitrusBR, 2010. 137 p.

PARENT, L. E. Diagnosis of the nutrient compositional space of fruit crops. Revista Brasileira de Fruticultura, v. 33, n. 1, p. 321-334, 2011.
PARTELLI, F. L. et al. Nutritional diagnosis of the organic conilon coffee trees (Coffea canephora Pierre ex Froehn): suffiency range approach for leaves and soil. Coffee Science, v. 1, n. 1, p. 43-49, 2006a.

PARTELLI, F. L. et al. Estabelecimento de normas DRIS em cafeeiro conilon orgânico ou convencional no Estado do Espírito Santo. Revista Brasileira de Ciência do Solo, v. 30, n. 1, p. 443-451, 2006b.

QUAGGIO, J. A. et al. Sources and rates of potassium for sweet orange production. Scientia Agricola, v. 68, n. 1, p. 369-375, 2011.

QUAGGIO, J. A. et al. Fruit yield and quality of sweet oranges affected by nitrogean, phosphorus, and potassium fertilization in tropical soils. Fruits, v. 61, n. 1, p. 1-10, 2006.

QUAGgIO, J. A. et al. Manejo da fertilidade do solo na citricultura. In: MATTOS JUNIOR, D. et al. Citrus. Campinas: Instituto Agronômico de Campinas, 2005. cap. 12, p. 483-507.

SANTANA, J. G. et al. Normas DRIS para interpretação de análises de folha e solo, em laranjeira pêra, na região central de Goiás. Pesquisa Agropecuária Tropical, v. 38, n. 2, p. 109-117, 2008.

SANTANA, J. G. et al. Estado nutricional da laranja pêra na região central do estado de Goiás avaliada pelas análises foliar e do solo. Bioscience Journal, v. 23, n. 3, p. 40-49, 2007.

SERRA, A. P. et al. Determinação de faixas normais de nutrientes no algodoeiro pelos métodos CHM, CND e DRIS. Revista Brasileira de Ciência do Solo, v. 34, n. 1, p. 105-113, 2010.

SILVA, F. A. S.; AZEVEDO, C. A. V. Versão do programa computacional assistat para o sistema operacional windows. Revista Brasileira de Produtos Agroindustrial, v. 4, n. 1, p. 71-78, 2002.

SISTEMA DE PROTEÇÃO DA AMAZÔNIA. Boletim climático da Amazônia. Manaus: Divisão de metereologia, 2005. 2 p.

URANO, E. O. M. et al. Determinação de teores ótimos de nutrientes em soja pelos métodos chance matemática, sistema integrado de diagnose e recomendação e diagnose da composição nutricional. Revista Brasileira de Ciência do Solo, v. 31, n. 1, p. 63-72, 2007.

WADT, P. G. S. Relationships between soil class and nutritional status of coffe cropes. Revista Brasileira de Ciência do Solo, v. 29, n. 1, p. 227-234, 2005.

WADT, P. G. S. et al. Fórmulas DRIS para o diagnóstico nutricional de pomares de cupuaçueiros. Bragantia, v. 70, n. 3 , p. 649-656, 2011.

WADT, P. G. S. et al. Valores de referência para macronutrientes em eucalipto obtidos pelos métodos DRIS e chance matemática. Revista Brasileira de Ciência do Solo, v. 34, n. 1, p. 685-692, 1998.

WADT, P. G. S.; SILVA, D. J. Acurácia do diagnóstico nutricional de pomares de mangueiras obtido por três fórmulas DRIS. Pesquisa Agropecuária Brasileria, v. 45, n. 10, p. 1180-1188, 2010.

WALWORTH, J. L.; SUMNER, M. E. The diagnosis and recommendation integrated system (DRIS). Advances in Soil Sciences, v. 12, n. 6, p. 149-188, 1987. 